\author{
Brief article
}

\title{
What does syntax say about space? 2-year-olds use sentence structure to learn new prepositions
}

\author{
Cynthia Fisher *, Stacy L. Klingler, Hyun-joo Song \\ Department of Psychology, University of Illinois, 603 E. Daniel Street, Champaign, IL 61820, USA \\ Received 31 August 2005; accepted 10 October 2005
}

\begin{abstract}
Children as young as two use sentence structure to learn the meanings of verbs. We probed the generality of sensitivity to sentence structure by moving to a different semantic and syntactic domain, spatial prepositions. Twenty-six-month-olds used sentence structure to determine whether a new word was an object-category name (This is a corp!) or a spatial-relational term (This is acorp my box!). We argue that children rely on the intimate relationship between nouns in sentences and semantic arguments of predicate terms: Noting that a new word takes noun arguments identifies the new word as a predicate term, and directs the child's attention to relations among its arguments. (C) 2005 Elsevier B.V. All rights reserved.
\end{abstract}

Keywords: Language acquisition; Word learning; Syntactic bootstrapping

Children use syntactic knowledge to learn verbs; this is known as syntactic bootstrapping (Landau \& Gleitman, 1985). Many studies have shown that young children assign different interpretations to verbs presented in different sentence structures (Fisher, 1996; 2002; Fisher, Hall, Rakowitz, \& Gleitman, 1994; Naigles, 1990; 1996; Naigles \& Kako, 1993). But how does syntactic bootstrapping begin?

We have proposed a 'structure-mapping' account of early syntactic bootstrapping, in which a shallow analysis of the sentence in which a new verb appears guides its interpretation (Fisher, 1996; 2002; Fisher et al., 1994; Gillette, Gleitman, Gleitman, \& Lederer, 1999). In essence, children use the number of nouns occurring with the new verb as a cue to its semantic predicate-argument structure. Transitive verbs license two noun

\footnotetext{
* Corresponding author. Tel.: +1 217333 3545; fax: +1 2172445876.

E-mail address: clfishe@uiuc.edu (C. Fisher).
} 
phrase arguments, and describe semantic relations between the two named entities. Intransitive verbs license one noun phrase, and denote an activity or state involving a single entity. Once children can identify some nouns, they can assign different interpretations to transitive and intransitive verbs simply by counting the nounsmapping a two noun sentence onto a conceptual predicate involving the two named entities, and a one noun sentence onto a conceptual predicate involving the single named entity.

The central assumption of the structure-mapping account of early syntactic bootstrapping is that children expect each referential term to be a semantic argument of a predicate term, and, conversely, expect the key semantic arguments of each predicate term to be spelled out by referential terms. This preference for a one-to-one mapping between referential terms in sentences and semantic arguments of predicate terms has been formalized in linguistic theory as the joint effects of the theta criterion and the projection principle (Chomsky, 1981).

The simple character of this one-to-one mapping preference makes a strong prediction. The number of nouns in a sentence should guide the interpretation not only of verbs, but of any argument-taking predicate. When a child notes that a word predicts some number of nouns in a sentence, this tells the child that the item is an argument-taking predicate, not whether it is a verb (Alice followed Bill), adjective (I'm happy for you), or preposition (The cat's on the mat). Although these constitute different grammatical categories in English, all take noun phrase arguments, and all encode semantic relations among the referents of those arguments. All should initially be interpretable in the way described above for verbs-by mapping each sentence onto a conceptual structure involving the participants named in the sentence.

The present study tested this prediction, asking whether young children would use sentence structures to learn the meanings of predicate terms other than verbs, namely, prepositions. Our research built on experiments by Landau and Stecker (1990) in which an experimenter placed an object on one corner of a box and uttered a new word as a noun (1) or a preposition (2). Syntactic context affected 3- and 5-year-olds' extension patterns. Children who heard (1) interpreted the word as a category name for an object: they rejected objects differing in shape from the original, but ignored location. Children who heard (2) interpreted the new word as a spatial relational term: they rejected locations not on the box, but ignored shape.

(1) This is a corp!

(2) This is acorp my box!

This task reveals the intimate relationship between noun-phrase arguments and semantic predicate-argument structure. Adding a noun after a new word told preschoolers that it must be a predicate term; observation of the event suggested that it described a spatial relation. The structure-mapping algorithm predicts that even very young children should be able to draw this inference: they should interpret the two nouns in a sentence describing a spatial array as evidence that the sentence conveys some relation between the referents of those two nouns. We tested this prediction with 2-year-olds, the earliest age 
at which children have been shown to use syntactic evidence to differentiate transitive from intransitive verbs (Naigles, 1990).

The children were tested in a looking-preference version of Landau and Stecker's (1990) task. For example, children saw a series of training events in which the new word (a) corp was taught: in each training trial, a hand placed a different duck on a box, and pointed to it as the new word was presented. Half of the children heard the new word presented as a noun ("This is a corp!"); half heard the new word presented as a preposition ("This is acorp my box!"). Following this familiarization, children saw two test events: one showed another duck beside the box (Object match), and the other showed a different object (e.g. eyeglasses) on the box (Location match). Children in the noun condition should interpret the word as a category name for an object, and therefore look longer at the object match; children in the preposition condition should interpret the new word as a relational term, and therefore look longer at the location match ${ }^{1}$.

\section{Method}

\subsection{Participants}

Forty-eight 2-year-olds (24 girls, 24 boys; $M=25.8$ months, range 24.6-27.0) participated. Nine additional children were eliminated due to fussiness (2), inattentiveness (1), side-bias (2), experimenter error (2), or because their vocabulary was more than 2 SD lower than the mean of the included children (2). Children's productive vocabularies, measured by the short form of the MacArthur CDI, ranged from 19 to 100, with a median of 66. These values are consistent with norms for this measure: Fenson, Pethick, Renda, Cox, Dale and Reznick (2000) reported a fiftieth-percentile score at 25 months of 73 for girls, and 58 for boys.

\subsubsection{Apparatus}

Children sat on a parent's lap facing two $20^{\prime \prime}$ monitors $30^{\prime \prime}$ away. The screens were at child's eye level, separated by $12^{\prime \prime}$. Audio stimuli were presented centrally; a hidden camera recorded the child's eye movements. Parents wore opaque glasses.

\subsubsection{Materials}

Two color videos were shown side-by-side. The novel word (a)corp was presented in the context of ducks on a box; (a)pilk accompanied cups under a table. The objects were 12 plastic ducks and 12 cups, varying in color, detailed shape, and size, and eight distractor objects that were neither ducks nor cups; the same box or table appeared in all events. The soundtracks were naturally-produced sentences recorded by a female native English speaker.

\footnotetext{
${ }^{1}$ This task relies on the well-established tendency of children and adults to look at scenes or objects closely related to the language they hear (e.g. Naigles, 1990; Tanenhaus, Spivey-Knowlton, Eberhard, \& Sedivy, 1995).
} 


\subsubsection{Procedure}

Following Waxman and Booth (2003), we presented and tested each novel word in three phases, as shown in Fig. 1: training, contrast, and test.

The (a)corp sequence began when the box appeared on both screens and was named ("This is my box"). Three training trials followed: in each, a duck appeared in front of the box on each screen (4s); a red-gloved hand placed each duck on top of the box simultaneously on both screens (4s); on one screen, the hand pointed to the duck, and the experimental sentence played ("This is acorp (my box)"; $3 \mathrm{~s}$ ); then, on the other screen, the hand pointed at the other duck, and the experimental sentence played again (“... and this is acorp (my box)!" 3s). This training was repeated twice more with different ducks.

In the contrast phase, the hand placed a toy carrot beside the box on one screen, pointed to it, and negated the experimental sentence ("Oops, this is not acorp (my box)"; 5s). The hand then placed a new duck on the box on the other screen and repeated the experimental sentence ("Yay, this is acorp (my box)! What's acorp (my box)?" 10s). The contrast phase was designed to draw attention to object category and location, and also gave the child an opportunity to hear wording similar to the test question before the test trial.

During the test phase, a new duck appeared in front of the box on one screen and toy eye-glasses in front of the box on the other screen (4s). The hand simultaneously moved the duck next to the box, and the glasses onto the box (4s), then placed another duck on the box on both screens and pointed to them (4s). At this point a 13-s still-frame test trial began, accompanied by the test sentence ("This is acorp (my box). What else is acorp (my box)?"). The first test trial was followed by a second contrast and test trial with different ducks and distractor objects. The entire procedure was repeated for the other novel word, yielding a total of four test trials, two for each word. Each training, contrast, or test trial was followed by $1 \mathrm{~s}$ of black screen on both videotapes. Left-right position of the videos and order of the novel words were counterbalanced across children.

\subsubsection{Coding}

We coded where children looked (left, right, away) during test trials, frame by frame from silent video. Reliability was assessed for 12 randomly selected children; coders agreed on $97.3 \%$ of frames. We calculated looking times to the location match, as a proportion of looking times to either the location- or object-match screen.

\subsubsection{Pretest with 2.5- and 3-year-olds}

We pre-tested the materials in an object-choice task with 243 -year-olds $(M=38.7$ months, 36-42) and 24 2.5-year-olds $(M=29.3$ months, 27-33). The choice task duplicated the sequence described above, except that the events were produced online by an experimenter; children received two rather than three training trials for each word; children chose an object in each test trial; and children learned three novel words: the two described above as well as "(a)dax" accompanying turtles in a bowl. We analyzed how often children chose the location match, as a proportion of location- and object-match choices.

Consistent with the findings of Landau and Stecker (1990), 3-year-olds were strongly influenced by sentence context: those in the preposition condition chose location matches more often $(M=.65, \mathrm{se}=.08)$ than did those in the noun condition $(M=.29$, se $=.10$; 

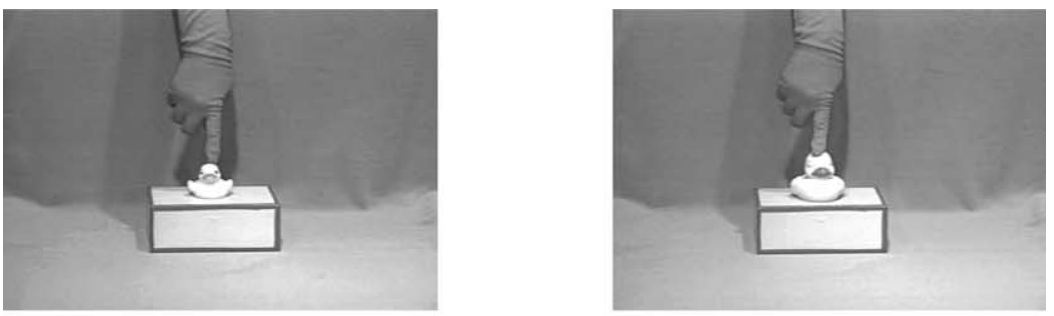

"This is acorp (my box) ... and this is acorp (my box)!"
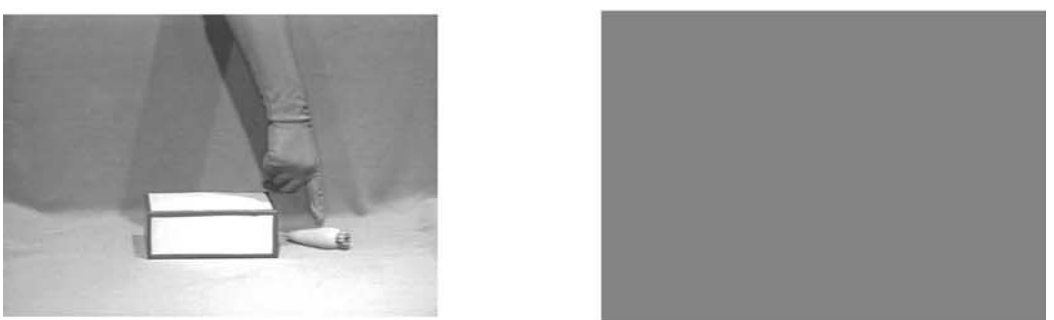

“Oops, this is not acorp (my box)!"
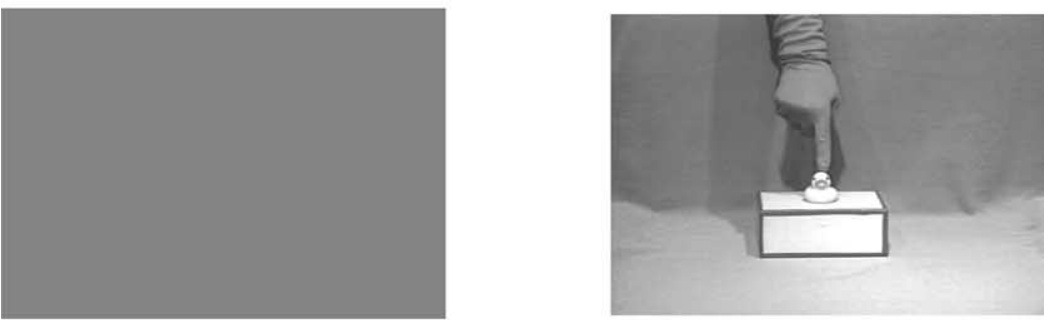

"Yay, this is acorp (my box)!

What's acorp (my box)? Can you find it?"

Object Match Screen

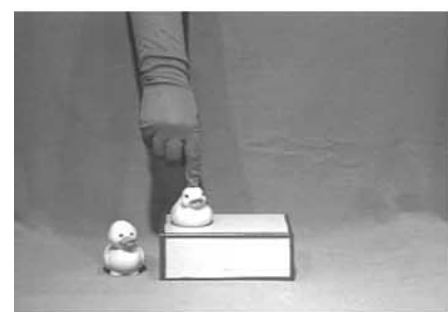

$\underline{\text { Location Match Screen }}$

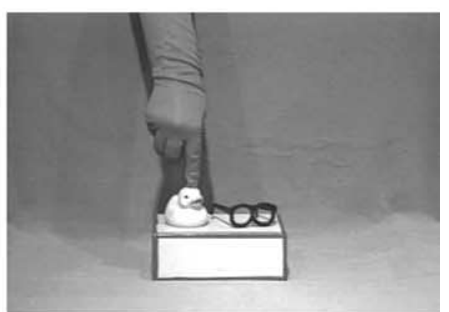

"This is acorp (my box). What else is acorp (my box)? Do you see it? What else is acorp (my box)? Can you find it?"

Fig. 1. Sample sequence of events in the preferential looking task. Each novel word was presented and tested in three phases: training (top row), contrast (second and third rows), and test (bottom row). 
$t(22)=2.90, P=.008)$. Among 2.5-year-olds, the effect of syntax depended on vocabulary (median $=75$, CDI short form). When the children were divided into high- and lowvocabulary groups, an ANOVA revealed an interaction of sentence and vocabulary $(F(1,20)=4.92, P=.038)$. Among high-vocabulary 2.5 -year-olds, those in the preposition condition chose location matches more often $(M=.74$, se $=.06)$ than did those in the noun condition $(M=.42$, se $=.11 ; t(10)=2.76, P=.02)$; low-vocabulary 2.5 -year-olds' choices did not depend on sentence context $(t<1)$.

As predicted, 3-year-olds and (higher-vocabulary) 2.5-year-olds who encountered a new word in a familiar labeling phrase (This is a corp) interpreted the word as an object name, and chose new examples of the same kind at test. Children who heard the new word followed by a familiar noun (This is acorp my box) interpreted the word as encoding a relationship between the figure and ground objects in the scene, and chose an object in the same relation to the ground object, regardless of kind. Among 2.5-year-olds we also found a relationship between vocabulary level and sensitivity to sentence context, suggesting that children's facility in detecting linguistic cues during the experiment could be roughly predicted by their vocabulary. We return to this issue in Section 3.

The performance of the low-vocabulary 2.5 -year-olds motivated us to reduce the number of novel words and increase the number of training trials for 2-year-olds. Despite these efforts, we considered it possible that a similar vocabulary effect might emerge for these younger children.

\section{Results}

Fig. 2 shows the average proportion of time spent looking at the location match screen, out of looking-time to either screen, for 2-year-olds whose productive vocabulary was above or below the median. The effect of sentence structure depended on vocabulary.

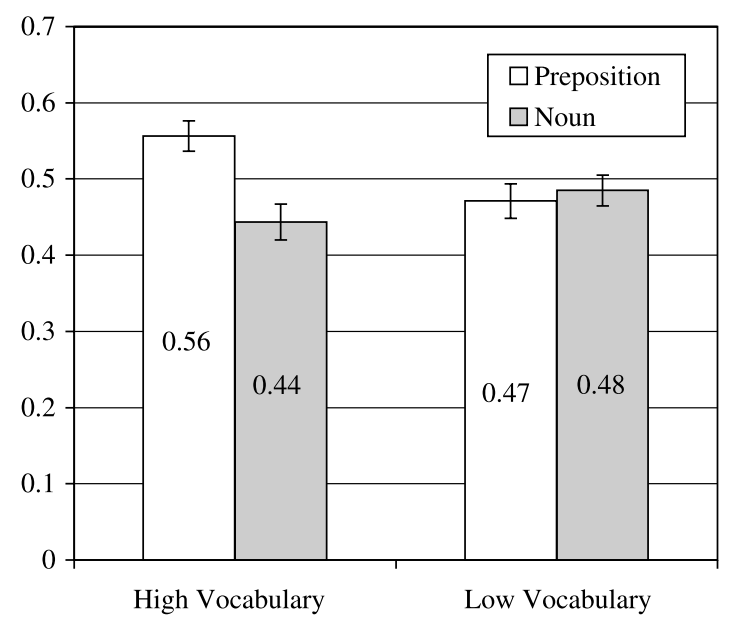

Fig. 2. The average (SE) proportion of time spent looking at the location match screen, out of looking-time to either screen, for 2-year-olds whose productive vocabulary was above or below the median. 
High-vocabulary 2-year-olds showed the predicted pattern: those in the preposition condition looked longer at the location match screen, whereas those in the noun condition looked longer at the object match screen.

This pattern was supported by an ANOVA on the proportion of time spent looking at the location match, yielding a main effect of sentence context $(F(1,44)=4.98, P=.03)$, no main effect of vocabulary $(F<1)$, and an interaction of sentence with vocabulary $(F(1,44)=8.14, P=.007)$. Among high-vocabulary 2-year-olds, those in the preposition condition looked longer at the location match than did those in the noun condition $(t(22)=3.55, P=.002)$. Looking patterns for low-vocabulary 2-year-olds did not vary with sentence condition $(t(22)<1)$. High-vocabulary 2-year-olds in the preposition condition looked longer at the location match than expected by chance $(.50, t(10)=2.82$, $P=.018)$; those in the noun condition looked longer at the object match than expected by chance $(t(12)=2.38, P=.035)$.

The effect of vocabulary was more obvious in the preposition than the noun condition. Within the preposition condition, high-vocabulary children looked longer at the location match than did low-vocabulary children (see Fig. $2 ; t(22)=2.77, P=.011$ ), while the preferences of children in the noun condition did not differ with vocabulary level $(t(22)=$ $1.30, P=.208)$. The noun-condition group, including high- and low-vocabulary children, looked longer at the object match than expected by chance $(M=.54$, se $=.02, t(23)=2.32$, $P=.029)$.

\subsection{Additional results}

Because our materials were naturally-produced sentences, the novel words differed across conditions in their sound patterns as well as in their syntactic context. Before we can attribute our results to syntactic context, we need to address the possibility that phonological cues led the children to interpret the novel nouns and prepositions differently. The novel words were sentence-final in the noun but not the preposition condition; therefore, they were lengthened in the noun context. English nouns are often sentence-final, and thus undergo final lengthening more often than words of other categories; consequently, lengthened words acoustically resemble nouns, while shorter words resemble words of other categories, including prepositions (Kelly, 1992). Moreover, in the noun condition the speaker intended to produce a determiner and a noun (e.g. a corp), while in the preposition condition she uttered a two-syllable word (acorp). The phonetic realization of a sequence is affected by the location of (prosodic) word boundaries (Fougeron \& Keating, 1997; Turk \& Shattuck-Hufnagel, 2000); the word boundaries intended by the speaker could have been marked by acoustic cues useful to the children (Gout, Christophe, \& Morgan, 2004).

To isolate the effect of sentence structure, we tested a new group of 16 2-year-olds $(M=26.0$ months, 24.8-26.9, median vocabulary=63) in an edited-noun condition. Sentences were created by deleting the noun phrase following each novel word in the original preposition-condition materials (e.g. This is acorp my box $\rightarrow$ This is acorp). The editing was done using Praat software, imposing silences beginning at zero-crossings in the waveforms to avoid obvious artifacts. The original recordings were of slow, careful speech, with care taken to release the novel words' final consonants; therefore, 
the resulting sentences sounded fairly natural. Children were tested just as in the main experiment.

Children in the edited-noun condition, like those in the original noun condition, looked longer at the object match than expected by chance $(M=.56$, se $=.02 ; t(15)=3.10$, $P=.007)$. An ANOVA comparing these data to those from the preposition condition again revealed a main effect of sentence context on the proportion of time spent looking at the location match $(F(1,36)=8.76, P=.005)$. The interaction of vocabulary and condition was not significant in this analysis $(F(1,36)=2.59, P=.117)$, but again the difference between the preposition and edited-noun conditions was reliable for high-vocabulary $(t(17)=3.06, P=.007)$ but not low-vocabulary 2-year-olds $(t(19)=1.01, P=.325)$. Thus, the same recorded novel words were interpreted differently depending on their syntactic context.

\section{Discussion}

Like the older children in our pretest and in Landau and Stecker's (1990) experiments, high-vocabulary 2-year-olds used sentence structure to interpret new words. Children in the noun condition used the familiar labeling phrase to identify the new word as a noun, and thus looked longer at the object match. They did so even when they heard novel words originally recorded as prepositions. Children in the preposition condition used the presence of a noun phrase following the new word to identify it as a predicate term, and thus looked longer at the location match.

Why did only high-vocabulary children show this effect, both among 2-year-olds in the main experiment and 2.5-year-olds in the pretest? There are at least two possibilities. One is that children need a vocabulary of a certain size or composition to attain the syntactic knowledge required to use sentence structure cues in word interpretation (Hirsh-Pasek \& Golinkoff, 1996). Another possibility is that vocabulary can serve as an index of languageprocessing facility. High-vocabulary children are faster and more accurate in retrieving lexical knowledge from memory (Fernald, Swingley, \& Pinto, 2001; Gershkoff-Stowe, 2002). This difference should affect performance in our task: to profit from sentence structure cues, children must quickly identify multiple words in the same sentence.

The present data do not provide strong reason to decide in favor of either account of the vocabulary effect. However, we can offer one recent source of evidence for the languageprocessing facility hypothesis. Yuan, Fisher, and Snedeker (in preparation) found that only high-vocabulary 26-month-olds interpreted transitive and intransitive verbs differently in a simple verb-learning task; when the pace of the sentences was slowed down, however, even 21-month-olds, with substantially lower vocabulary scores, succeeded at the task. This finding suggests that the vocabulary effect in the older group was due, not to the need for any particular level of vocabulary development, but to higher-vocabulary children's ability to more quickly and accurately process sentences. Ongoing investigations further test this account by simplifying experimental materials for even younger and less linguistically sophisticated children. 


\subsection{Consequences for syntactic bootstrapping}

The present findings extend the syntactic and semantic scope of investigations of syntactic bootstrapping. Previous studies documented that 2 -year-olds prefer to interpret transitive but not intransitive verbs as referring to animate causal or contact action (Fisher, 2002; Naigles, 1990; Naigles \& Kako, 1993). The present research shows that children find syntax informative in a different syntactic and semantic domain: 26-month-olds can use syntax to guide interpretation of a novel predicate term, whether it is a verb or a preposition, and whether the nouns in the sentence refer to participants in an action event or to objects in a spatial array. These findings suggest a quite general sensitivity to the argument-licensing powers of predicate terms.

The syntactic and semantic flexibility of early sensitivity to sentence structure is just what we should expect if young children have access to the structure-mapping procedure we sketched in the Introduction. Noting that a new word predicts the occurrence of nouns in sentences identifies the word as a predicate term, and provides evidence relevant to its semantic predicate-argument structure. The structure-mapping procedure depends only on the number of nouns in the sentence; it therefore provides no initial mechanism for telling apart what will ultimately be different syntactic classes of argument-taking predicates.

This lack of specificity may seem like a disadvantage - the number of nouns in a sentence clearly provides only a very abstract guide to the interpretation of predicate terms. We suggest, however, that this constraint is abstract enough to guide early comprehension despite variation among languages in relations between syntax and semantics. Not all languages possess categories of prepositions and predicate adjectives that are distinct from verbs; they may instead use main verbs to convey spatial or property meanings (Croft, 1990; Maratsos, 1990). The simple structure-mapping procedure allows languages to vary in the partitioning of predicate meanings across grammatical categories, yet provides helpful constraints on initial sentence interpretation (see Waxman, 2004, for a similar argument).

Learners of English must eventually separate verbs, prepositions, and adjectives into distinct grammatical categories. Children could accomplish this separation in part by detecting the function morphemes and meanings characteristic of each category (Gerken \& McIntosh, 1993; Maratsos, 1982; 1990; Mintz, 2002; Waxman \& Booth, 2003). Santelmann and Jusczyk (1998) reported that 18-month-olds detect the dependency between is and -ing in verb phrases; children also begin to learn spatial prepositions early in the second year (Casasola \& Wilbourn, 2004; Meints, Plunkett, Harris, \& Dimmock, 2002). Such findings reveal hints of the morphological and semantic knowledge relevant to disentangling verbs from prepositions. Once a new word has been identified as a predicate term based on its argument-taking properties, its morphological markings-in the present case, the absence of verb morphology — could provide additional constraints on its interpretation. Further investigations of toddlers' knowledge of verb-phrase morphology will be needed to discover when and how children discriminate prepositions from verbs and develop separate expectations for their meanings.

The structure-mapping proposal suggests that children approach the language-learning task expecting a fundamental grammatical distinction between referential and relational terms (Lidz \& Gleitman, 2004; Maratsos, 1990; Osgood, 1980). Verbs and other predicate 
terms signal their relational semantics by their combinatorial behavior: they license the appearance of referential terms in sentences, and thus betray their relational semantics even to the youngest observer.

\section{Acknowledgements}

This research was partially funded by NIH grant HD44458, and by the Research Board of the University of Illinois. We thank Renée Baillargeon, Jennifer Cole, Yael Gertner, and Sylvia Yuan for helpful comments.

\section{References}

Casasola, M., \& Wilbourn, M. (2004). Fourteen-month-old infants can form novel word-spatial relation associations. Paper presented at the International Conference on Infant Studies, Chicago.

Chomsky, N. (1981). Lectures on government and binding. Dordrecht: Foris.

Croft, W. (1990). Typology and universals. Cambridge: Cambridge University Press.

Fenson, L., Pethick, S., Renda, C., Cox, J. L., Dale, P. S., \& Reznick, J. S. (2000). Short-form versions of the MacArthur communicative development inventories. Applied Psycholinguistics, 21, 95-115.

Fernald, A., Swingley, D., \& Pinto, J. P. (2001). When half a word is enough: Infants can recognize spoken words using partial phonetic information. Child Development, 72, 1003-1015.

Fisher, C. (1996). Structural limits on verb mapping: The role of analogy in children's interpretations of sentences. Cognitive Psychology, 31, 41-81.

Fisher, C. (2002). Structural limits on verb mapping: The role of abstract structure in 2.5-year-olds' interpretations of novel verbs. Developmental Science, 5, 55-64.

Fisher, C., Hall, D. G., Rakowitz, S., \& Gleitman, L. (1994). When it is better to receive than to give: Syntactic and conceptual constraints on vocabulary growth. Lingua, 92, 333-375.

Fougeron, C., \& Keating, P. A. (1997). Articulatory strengthening at edges of prosodic domains. The Journal of the Acoustical Society of America, 101, 3728-3740.

Gerken, L., \& McIntosh, B. J. (1993). Interplay of function morphemes and prosody in early language. Developmental Psychology, 29, 448-457.

Gershkoff-Stowe, L. (2002). Object naming, vocabulary growth, and the development of word retrieval abilities. Journal of Memory and Language, 46, 665-687.

Gillette, J., Gleitman, H., Gleitman, L., \& Lederer, A. (1999). Human simulations of vocabulary learning. Cognition, 73, 135-176.

Gout, A., Christophe, A., \& Morgan, J. L. (2004). Phonological phrase boundaries constrain lexical access II. Infant data. Journal of Memory and Language, 51, 548-567.

Hirsh-Pasek, K., \& Golinkoff, R. (1996). The origins of grammar. Cambridge, MA: MIT Press.

Kelly, M. H. (1992). Using sound to solve syntactic problems: The role of phonology in grammatical category assignments. Psychological Review, 99, 349-364.

Landau, B., \& Gleitman, L. R. (1985). Language and experience: Evidence from the blind child. Cambridge, MA: Harvard University Press.

Landau, B., \& Stecker, D. (1990). Objects and places: Geometric and syntactic representations in early lexical learning. Cognitive Development, 5, 287-312.

Lidz, J., \& Gleitman, L. R. (2004). Yes, we still need universal grammar. Cognition, 94, 85-93.

Maratsos, M. (1982). The child's construction of grammatical categories. In E. Wanner, \& L. R. Gleitman (Eds.), Language acquisition: The state of the art (pp. 240-266). Cambridge: Cambridge University Press.

Maratsos, M. (1990). Are actions to verbs as objects are to nouns? On the differential semantic bases of form, class, category. Linguistics, 28, 1351-1379. 
Meints, K., Plunkett, K., Harris, P. L., \& Dimmock, D. (2002). What is 'on' and 'under' for 15-, 18- and 24month-olds? Typicality effects in early comprehension of spatial prepositions. British Journal of Developmental Psychology, 20, 113-130.

Mintz, T. H. (2002). Category induction from distributional cues in an artificial language. Memory and Cognition, 30, 678-686.

Naigles, L. (1990). Children use syntax to learn verb meanings. Journal of Child Language, 17, 357-374.

Naigles, L. (1996). The use of multiple frames in verb learning via syntactic bootstrapping. Cognition, 58, 221-251.

Naigles, L., \& Kako, E. T. (1993). First contact in verb acquisition: Defining a role for syntax. Child Development, 64, 1665-1687.

Osgood, C. E. (1980). Lectures on language performance. New York: Springer.

Santelmann, L. M., \& Jusczyk, P. W. (1998). Sensitivity to discontinuous dependencies in language learners: Evidence for limitations in processing space. Cognition, 69, 105-134.

Tanenhaus, M. K., Spivey-Knowlton, M. J., Eberhard, K. M., \& Sedivy, J. C. (1995). Integration of visual and linguistic information in spoken language comprehension. Science, 268, 1632-1634.

Turk, A. E., \& Shattuck-Hufnagel, S. (2000). Word-boundary-related duration patterns in English. Journal of Phonetics, 28, 397-440.

Waxman, S. (2004). Everything had a name, and each name gave birth to a new thought: Links between early word learning and conceptual organization. In D. G. Hall, \& S. R. Waxman (Eds.), Weaving a lexicon (pp. 295-335). Cambridge, MA: MIT Press.

Waxman, S., \& Booth, A. (2003). The origins and evolution of links between word learning and conceptual organization: New evidence from 11-month-olds. Developmental Science, 6, 128-135. 